\title{
Microwave-Assisted Modification of Carbon Nanotubes with Biocompatible Polylactic Acid
}

\author{
Qi Zhang, Shijun Zhang, Liying Zhang \\ Beijing Research Institute of Chemical Industry, SINOPEC, Beijing, China \\ Email: zhangqi01.bjhy@sinopec.com
}

Received October 2013

\begin{abstract}
Polylactic acid (PLA) was successfully covalently grafted onto multi-walled carbon nanotubes (MWCNT) by microwave-assisted polymerization of lactide monomers. The final products MWCNT-g-PLA were characterized with Fourier-transform IR (FTIR), Raman spectroscopy, thermogravimetric analyses (TGA) and transmission electron microscopy (TEM). The results indicated PLA chain was covalently attached to the MWCNT. The grafted PLA was uniformly coated on the surface of MWCNT with a layer thickness of $2 \sim 6 \mathrm{~nm}$. The grafted PLA content could be controlled by microwave irradiation time and the concentrations of reactant. The product with $60.5 \%$ grafted PLA content can be synthesized in one hour.
\end{abstract}

\section{KEYWORDS}

Multi-Walled Carbon Nanotubes; Polylactic Acid; Microwave; Biocompatible

\section{Introduction}

Since the discovery of carbon nanotubes (CNTs) in the early 1990 [1], their unique atomic structure, very high aspect ratio and outstanding physical and chemical properties have attracted great attention and imagination of many scientists. As known to all, carbon nanotubes possess high flexibility, low mass density, and large aspect ratio (typically $>1000$ ), whereas they present extraordinary high tensile strength and modulus, together with excellent electrical properties [2,3]. The unique properties of CNT offered many opportunities for their applications such as reinforcement of fibers and nanocomposites, field-emission displays and nanosize probe tips for atomic force microscopy. However, the super amphiphobic property of carbon nanotubes is still the fundamental and technical barriers toward expanding many of the applications [4]. The inherent insolubility and aggregation of CNT in polymer composites prevent efficient stress from transferring to individual nanotubes and have a bad effect on efficiency usage of its properties [5]. Therefore, many researches on covalent and noncovalent modification of CNT have been made to realize the well dispersion of individual nanotubes and establish a strong chemical affinity with the surrounding polymer matrix [6-8]. The noncovalent approaches include surfactant modification [9-14], polymer wrapping, [15,16] and polymer absorption $[17,18]$. The covalent functionaliza- tion of CNT is usually realized by grafting polymer or long alkyl chains onto CNT [19-23].

Since the environmental pollution and the exhaustion of petroleum resources become critical issues, Polylactic acid (PLA) has been spotlighted as a biodegradable, sustainable and eco-friendly substituent for petroleum-based polymers [24,25]. Accordingly, the combination of PLA with CNT will broaden the novel application of carbon nanotubes. Several researchers have prepared PLAfunctionalized CNTs via "grafting from" and/or "grafting to" methods. Chen et al. [26,27] synthesized PLA functionalized CNTs by reacting functional group $\mathrm{COCl}$ on the surface of CNTs with different weight molecular PLA or using the surface initiating ring-open polymerization of L-lactide. Song et al. have prepared CNT-gPLAs by polycondensation of L-lactic acid with carboxylic acid-functionalized CNT in xylene solution. [28] Feng et al. reported that the PLA was covalently grafted onto the surface of magnetic multiwalled carbon nanotubes (m-MWCNTs) by in-situ ring-opening polymerization of lactide [21]. However, the conventional heating route to prepare the PLA grafted carbon nanotubes required strict conditions (vacuum, high temperature and long reaction time) which are obviously not good for commercially application.

In this paper, we present microwave-assisted method to realize the grafting of PLA onto multi-wall carbon 
nanotubes (MWCNT). The MWCNT was first treated by acid to gain an active $\mathrm{COOH}$ group on the surface, and then acid-functionalized MWCNT (MWCNT-COOH) induced in-situ polycondensation of lactide together with $\mathrm{SnOct}_{2}$. The grafting and polymerization rate were much faster than that of conventional heating. We investigated the morphology and structure of the final grafting product. In addition, the influences of reaction conditions were discussed further.

\section{Experimental Section}

\subsection{Materials}

The multi-walled carbon nanotubes (MWCNTs) (>98\%, with an outside diameter of 20 - $30 \mathrm{~nm}$, an inside diameter of $5-10 \mathrm{~nm}$, and lengths of $10-30 \mathrm{~mm}$ ) were purchased from Cheaptubes Co.Ltd, USA, and used as recived. D, L-Lactide (Aldrich) and Stannous octoate (Sn(Oct) $)_{2}$, 98\%, Sinopharm Chemical Reagent Co. Ltd.) were used as received. Sulfuric acid, nitric acid, methanol, chloroform, and N, N-dimethylformatide (DMF) as well as other chemicals were purchased from Aldrich and used without further purification.

\subsection{Acid Treatment of MWCNT}

The acid-functionalized multi-wall carbon nanotube (MWCNT-COOH) was prepared from the pristine MWCNT as the previous report [29]. MWCNTs were treated with a mixture of concentrated sulfuric and nitric acids (volume 3:1). The mixture was ultrasonicated (power of $60 \mathrm{~W}$ and nominal frequency of $40 \mathrm{kHz}$ at ambient temperature) for $30 \mathrm{~min}$, and then magnetically stirred at $80^{\circ} \mathrm{C}$ for $4 \mathrm{~h}$. After the mixture was cooled to room temperature, it was diluted with deionized water and then vacuum-filtered through $0.22 \mathrm{~mm}$ Millipore polypropylene membrane, and washed with distilled water until the $\mathrm{pH}$ value was moderate ( $\sim$ ca. 7$)$. The filtered product was dried under vacuum for $24 \mathrm{~h}$ at $60^{\circ} \mathrm{C}$, giving the acid-functionalized MWCNT-COOH. The amount of carboxylic acid groups of MWCNT-COOH prepared in this study was determined to be $0.0023 \mathrm{~mol}$ per $1.0 \mathrm{~g}$ MWCNT.

\subsection{Microwave-Assisted Functionalization of MWCNT-COOH with PLA}

The apparatus used for the polymerization was a domestic microwave oven (Meiling, China, $2450 \mathrm{MHz}$ and $800 \mathrm{~W}$ ). A mixture of purified MWCNT-COOH, Lactide and catalyst stannous chloride as well as a certain amount solvent DMF in an open beaker was first ultrasonicated for $30 \mathrm{~min}$ and then irradiated at the microwave power of $200 \mathrm{~W}$ for some period of time. Then the crude product was cooled to room temperature and washed several times by chloroform and precipitated in methanol, and the precipitate was dried under vacuum for $12 \mathrm{~h}$ at $60^{\circ} \mathrm{C}$ to gain the final product MWCNT- $g$-PLA.

\subsection{Characterization of MWCNT-g-PLA}

Fourier transform infrared (FTIR) spectra (collected from a Bruker Tensor 27 FTIR system) were used to characterize the molecular structure. The samples of nanocomposites were imbedded in $\mathrm{KBr}$ disks. Raman spectra used to confirm the structure of MWCNTs operating at $514 \mathrm{~nm}$ with a resolution of $1.5 \mathrm{~cm}^{-1}$. Transmission electron microscopy (TEM) images were examined using a FEI Tecnai-20 instrument operated at a $100 \mathrm{kV}$ accelerating voltage to observe the nanoscale structures of the various MWCNT-g-PLA. Thermogravimetric analysis (TGA) was conducted in nitrogen atmosphere. MWCNT and MWCNT-g-PLA were heated at a heating rate of $10^{\circ} \mathrm{C} / \mathrm{min}$ from $50^{\circ} \mathrm{C}$ to $500^{\circ} \mathrm{C}$, to determine the graft content of PLA.

\section{Result and Discussion}

PLA is grafted from the surface of MWCNT-COOH by the microwave-assisted ring opening polymerization of lactide monomers. It's reported that, in the pristine state, $\mathrm{Sn}(\mathrm{Oct})_{2}$ does not contain any reactive alkoxide groups and that the alcohol employed usually as coinitiator substitutes at least one of the octanoate groups in a rapid equilibration, and the resulting Sn alkoxide is then the true initiator of the polymerization process [18]. Therefore, in our study, the polymerization is initiated by $\mathrm{Sn}(\mathrm{Oct})_{2}$ together with hydroxyl which is actually contained by carboxylic acid group on the surface of MWCNT-COOH.

\subsection{FT-IR Spectra}

In our investigation, we focused on the structure analysis and characterization and the effect of reaction conditions on the grafting rate of final product MWCNT-g-PLA. The FTIR spectra of MWCNT and MWCNT-g-PLA were shown in Figure 1. The spectrum of MWCNT$\mathrm{COOH}$ presented a relatively weak peak at $1750 \mathrm{~cm}^{-1}$ which corresponds to the incorporated carboxylic acid groups as a result of the acid treatment process. After polymerization, the spectrum of MWCNT- $g$-PLA sample showed relatively strong absorption peaks around 1750 $\mathrm{cm}^{-1}$ assigned to the $\mathrm{C}=\mathrm{O}$ stretching, which clearly indicated that the PLA molecules were covalently bound to the MWCNTs.

\subsection{Raman Spectroscopy}

Raman spectroscopy is a powerful tool used for the characterization of functionalized CNTs. As shown in 


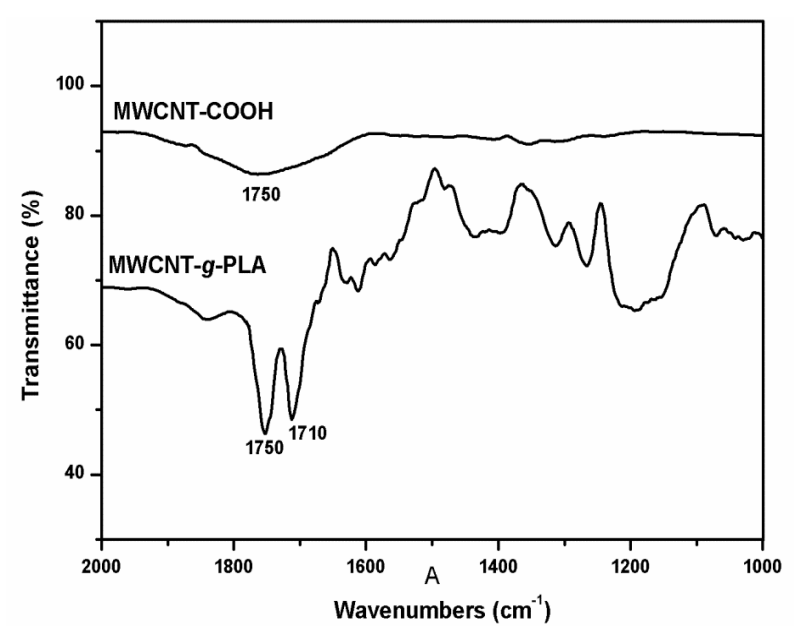

Figure 1. FTIR spectra of MWCNT-COOH and MWCNTg-PLA. The IR sample of MWCNT-g-PLA was prepared by irradiating at the microwave power of $200 \mathrm{~W}$ for $60 \mathrm{~min}$, $[\mathrm{LA}] /[\mathrm{COOH}]=20,[\mathrm{LA}] /\left[\mathrm{Sn}(\mathrm{Oct})_{2}\right]=2000$.

Figure 2, the D and G bands of the MWCNTs at 1285 and $1600 \mathrm{~cm}^{-1}$ which are attributed to the defects and disorder-induced peaks and tangential-mode peaks were clearly observed in both MWCNT-COOH and MWCNTg-PLA spectrums. The intensity of MWCNT-g-PLA was greatly decreased than that of MWCNT-COOH. Meanwhile, the Raman signals of neat PLA were not found in the spectrum of MWCNT-g-PLA. That result possibly can be related to the structure change of MWCNT [27]. After covalently fictionalization, the polymer chains grafted to the MWCNT surface formed nanometer-scale layer. The energy transfer between the MWCNT and PLA layer as well as the effect of grafted PLA chains on the electronic properties of the MWCNT induced the changes of the Raman signals.

\subsection{Morphologies}

A further certification of grafting PLA onto MWCNT was determined by transmission electron microscopy. The fine nanostructures of the as-prepared MWCNT-gPLA as well as the acid-functionalized CNT were shown in Figure 3. The images clearly show that the surface morphology of MWCNT-g-PLA is significantly different, by contrasting with MWCNT-COOH. The diameter marked in the image is $\sim 30 \mathrm{~nm}$. In the TEM images of MWCNT-COOH (Figure 3(a), (b)), the CNT wall was relatively smooth and clean and was not obviously covered with an extra phase. After grafting PLA from MWCNTs, a relatively rough polymer layer can be easily distinguished, with a thickness of $2 \sim 6 \mathrm{~nm}$. With high magnifications, it could be seen that a homogeneous shell had formed on all of the surface s of MWCNTs. Furthermore, the original length and pattern of MWCNTs remain intact after a series of chemical treatments, which

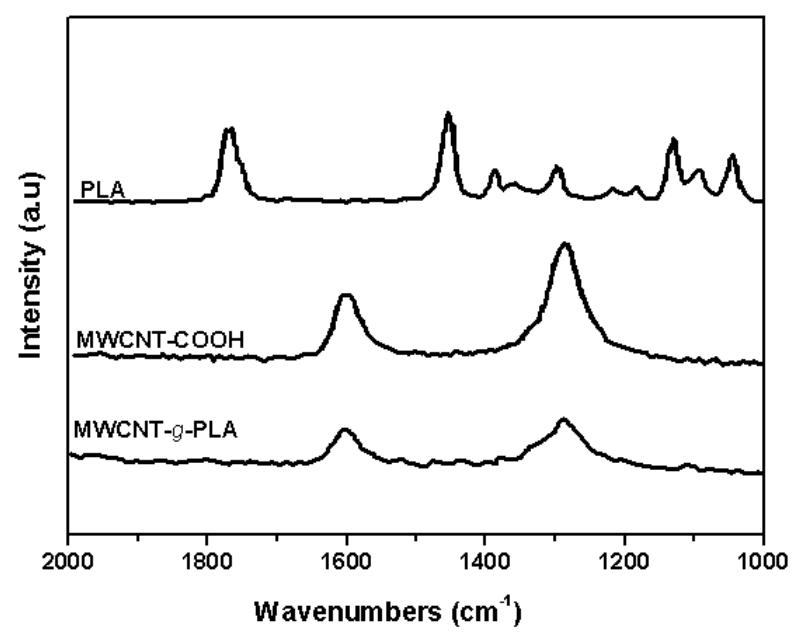

Figure 2. Raman spectra of PLA, MWCNT-COOH and MWCNT-g-PLA samples.
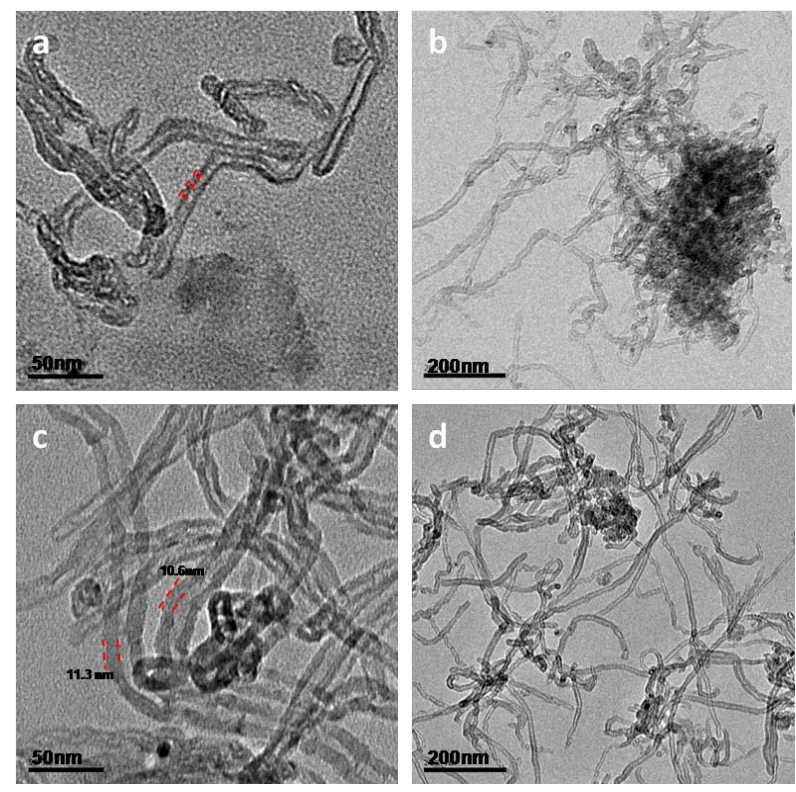

Figure 3. TEM images of MWCNT-COOH and MWCNTg-PLA at different magnifications. (a) MWCNT-COOH (high magnification), (b) MWCNT-COOH (low magnification), (c) MWCNT-g-PLA (high magnification) and (d) MWCNT-g-PLA (low magnification).

can be identified in Figure 3(d).

\subsection{Grafting Content of MWCNT-g-PLA}

To evaluate the content of grafted PLA, thermogravimetric analysis (TGA) of MWCNT-COOH and MWCNTg-PLA was performed in Figure 4. For MWCNT-COOH, more than $95 \mathrm{wt} \%$ was retained when heated over a temperature range of $50^{\circ} \mathrm{C} \sim 500^{\circ} \mathrm{C}$. However, the MWCNTg-PLA exhibited major weight loss in the temperature range of $300^{\circ} \mathrm{C} \sim 400^{\circ} \mathrm{C}$, this is approximately caused by the degradation of the PLA grafted on to the MWCNTs. 


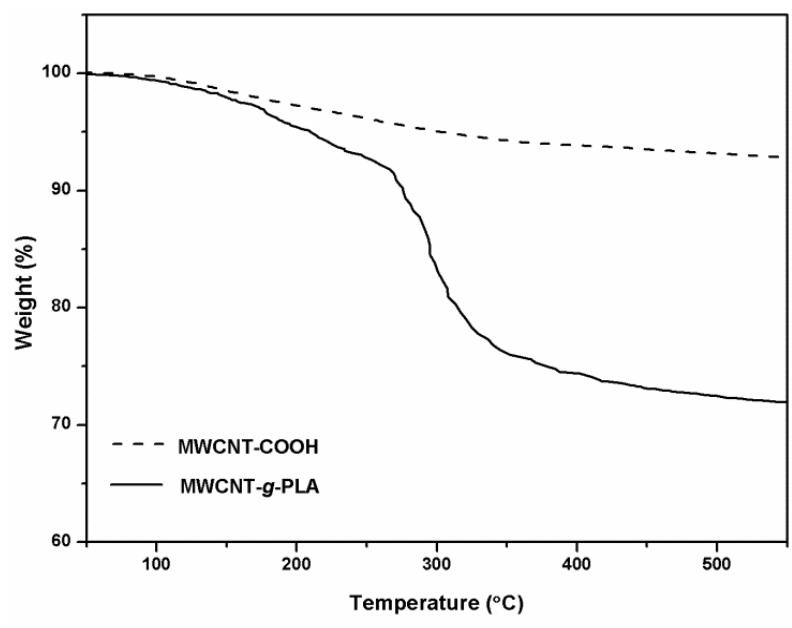

Figure 4. Thermogravimetric analysis (TGA) of MWCNTCOOH and MWCNT-g-PLA. The sample of MWCNTg-PLA was prepared by irradiating at the microwave power of $200 \mathrm{~W}$ for $45 \mathrm{~min},[\mathrm{LA}] /[\mathrm{COOH}]=20,[\mathrm{LA}] /\left[\operatorname{Sn}(\mathrm{Oct})_{2}\right]=$ 2000 .

Comparing the mass gain after the grafting reaction with the thickness of the PLA layer observed in the TEM images of the MWCNT-g-PLA samples, we proposed that the larger the amount of grafted PLA, the thicker the polymer shell. In the study, the degree of weight loss at $500^{\circ} \mathrm{C}$ in TGA was taken as the content of grafted PLA.

With the increasing reaction time, the gained PLA content presents an increasing tendency (Figure 5). Accordingly, by considering that MWCNT-COOH in this study possess carboxylic acid groups of $0.0023 \mathrm{~mol}$ for MWCNT of $1 \mathrm{~g}$ and by assuming that all the carboxylic acid groups of MWCNT-COOH are reacted with lactide during the ring-opening polymerization, the apparent number average molecular weights of grafted PLA chains of MWCNT-g-PLAs synthesized at various irradiation time could be evaluated, as can be seen in Table 1 and Figure 5. As shown in Figure 5 and Table 1, the PLA content was increased with the prolonged irradiation time; meanwhile the increasing tendency gradually became flat. The number-average molecular weight of grafted PLA chains could reach up to 1430 by only 90 minutes. According to the results, the fine products were obtained in a very short irradiation time. Different from conventional heating, the microwave-assisted modification process dismissed vacuum because of the accelerated reaction rate. Comparing the results under microwave irradiation with conventional heating method, it was considered that the enhanced polymerization rate originated not only from thermal effects but also from microwave effects.

\subsection{Effect of Reactant Ratio}

In order to further investigate the influence of reaction

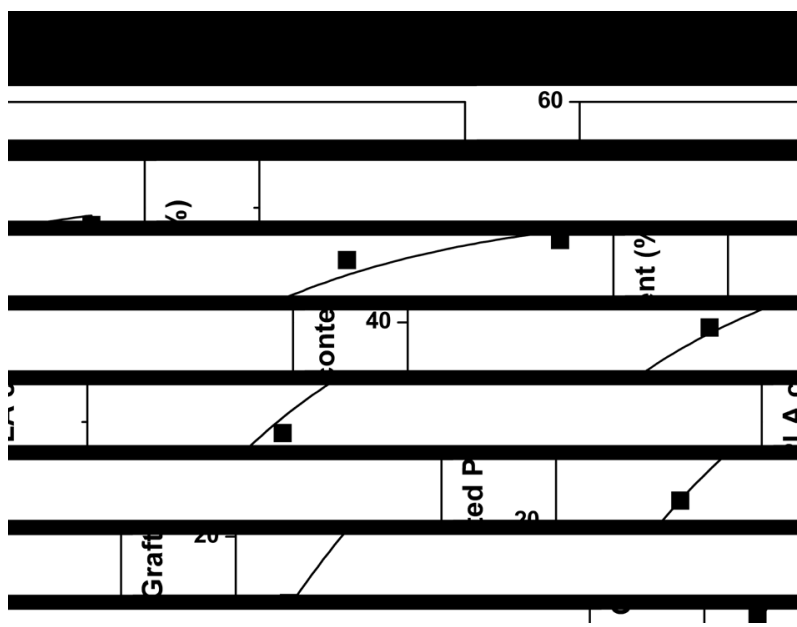

Figure 5. Changes of grafted PLA content with different microwave irradiation time. Condition: Microwave power $200 \mathrm{~W},[\mathrm{LA}] /[\mathrm{COOH}]=20,[\mathrm{LA}] /\left[\operatorname{Sn}(\mathrm{Oct})_{2}\right]=2000$.

Table 1. Weight contents and number average molecular weights of grafted PLA chains for MWCNT-g-PLA.

\begin{tabular}{ccc}
\hline $\begin{array}{c}\text { Irradiation } \\
\text { time (min) }\end{array}$ & $\begin{array}{c}\text { The grafted } \\
\text { PLA content }^{\mathrm{a}}(\%)\end{array}$ & $\begin{array}{c}\text { Number-average molecular } \\
\text { weight of the grafted PLA }\end{array}$ \\
\hline 10 & 12.1 & 240 \\
20 & 22.5 & 440 \\
30 & 28.7 & 675 \\
45 & 39.4 & 1100 \\
60 & 45.6 & 1350 \\
90 & 48.2 & 1430 \\
\hline
\end{tabular}

${ }^{\mathrm{a}}$ The grafted PLA content in MWCNT- $g$-PLA is evaluated from TGA thermograms. ${ }^{\mathrm{b}}$ The concentration of carboxylic acid groups is $0.0023 \mathrm{~mol}$ per MWCNT of $1.0 \mathrm{~g}$.

conditions on the final product, we also studied the variation of gained grafted PLA content in MWCNT-g-PLA which prepared by different catalyst concentrations. Figure 6 shows the effect of varied $\mathrm{Sn}(\mathrm{Oct})_{2}$ concentration and different $[\mathrm{LA}] /[\mathrm{COOH}]$ ratio on the grafted PLA content. The grafted polymer content in MWCNT-g-PLA was strongly dependent on the amount of $\mathrm{Sn}(\mathrm{Oct})_{2}$ and the ration of $[\mathrm{LA}] /[\mathrm{COOH}]$. As shown in Figure 6(a), with the increasing $\mathrm{Sn}(\mathrm{Oct})_{2}$ concentration from 0.01 to $0.2 \%$, the content of PLA in MWCNT-g-PLA increased until it reached a maximum at $0.05 \%$. However, the concentration of $\mathrm{Sn}(\mathrm{Oct})_{2}$ which is larger than $0.05 \mathrm{~mol} \%$ is not good for PLA grafting. This phenomena of inhibition can be explained by that $\mathrm{Sn}(\mathrm{Oct})_{2}$ catalyzes not only the polymerization of LA but also the decomposition of polymer. Too much $\mathrm{Sn}(\mathrm{Oct})_{2}$ might facilitated decomposition than polymerization. On the other hand, the reaction that PLA grafting to MWCNT was induced by active species that generated by carboxylic acid groups and 

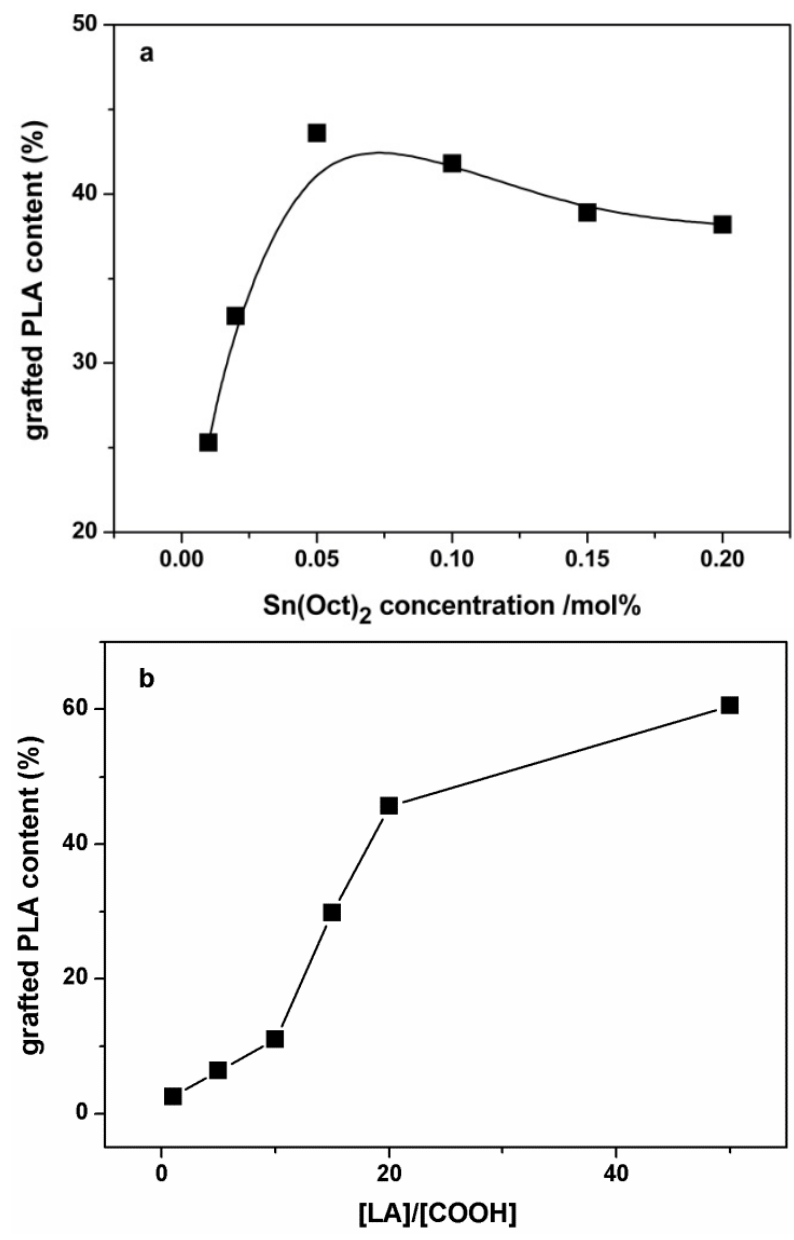

Figure 6 The effect of $\mathrm{Sn}(\mathrm{Oct})_{2}$ concentration and [LA]/ [COOH] ratio on the grafted PLA content. (a) Microwave power $200 \mathrm{~W}$, irradiation time $60 \mathrm{~min},[\mathrm{LA}] /[\mathrm{COOH}]=20$. (b) Microwave power $200 \mathrm{~W}$, irradiation time $60 \mathrm{~min}$, [LA]/ $\left[\mathrm{Sn}(\mathrm{Oct})_{2}\right]=\mathbf{2 0 0 0}$.

$\mathrm{Sn}(\mathrm{Oct})_{2}$, with fixed concentration of carboxylic acid groups, higher concentration of $\mathrm{Sn}(\mathrm{Oct})_{2}$ didn't increase the number of active species. The different ratio of [LA]/ $[\mathrm{COOH}]$ also has great influence on the grafted PLA content. The result (Figure 6(b)) suggested that larger ratio can generate higher grafted PLA content. With the $[\mathrm{LA}] /[\mathrm{COOH}]$ ratio of 50 , the gained PLA amount in MWCNT-g-PLA could reach up to $60.5 \%$. By controlling the reaction condition, we can get the final product with different grafting rate.

\section{Conclusion}

The biocompatible Polylactic acid were directly grafted to acid functionalized MWCNT by microwave irradiation. The final grafted product MWCNT-g-PLA with different PLA grafting rate can be synthesized in only 60 min. The TEM images of coated PLA layer clearly indicated the grafted PLA was uniform on the surface of
MWCNT. Moreover, the molecular weight and grafted content can be well controlled by altering $\mathrm{Sn}(\mathrm{Oct})_{2}$ concentration and irradiation time as well as $[\mathrm{LA}] /[\mathrm{COOH}]$ ratio. With the conditions which the microwave power is $200 \mathrm{~W}$ and the ratio of $[\mathrm{LA}] /[\mathrm{COOH}]$ and $[\mathrm{LA}] /\left[\mathrm{Sn}(\mathrm{Oct})_{2}\right]$ is 50 and 2000 respectively, the MWCNT-g-PLA which the grafted PLA chains could reach up to $60.5 \mathrm{wt} \%$ can be prepared in only one hour. Different from conventional heating, the microwave-assisted modification process dismissed vacuum because of the accelerated reaction rate. The microwave-assisted method shows the advantages of rapid speed and benign conditions over conventional heating and should be favored in industrialization for commercially biocompatible nanocomposites.

\section{REFERENCES}

[1] S. Iijima, "Helical Microtubules of Graphitic Carbon," Nature, Vol. 354, No. 6348, 1991, pp. 56-58. http://dx.doi.org/10.1038/354056a0

[2] Y. P. Sun, K. F. Fu, Y. Lin and W. I. Huang, "Functionalized Carbon Nanotubes: Properties and Applications," Accounts of Chemical Research, Vol. 35, No. 12, 2002, pp. 1096-1104. http://dx.doi.org/10.1021/ar010160v

[3] P. J. F. Harris, “Carbon Nanotube Composites,” International Materials Reviews, Vol. 49, No. 1, 2004, pp. 31-43. http://dx.doi.org/10.1179/095066004225010505

[4] F. J. Gomez, R. J. Chen, D. Wang, R. M. Waymouth and H. Dai, "Ring Opening Metathesis Polymerization on Non-Covalently Functionalized Single-Walled Carbon Nanotubes," Chemical Communications, Vol. 2, 2003, pp. 190-191. http://dx.doi.org/10.1039/b211194b

[5] R. Andrews and M. C. Weisenberger, "Carbon Nanotube Polymer Composites," Current Opinion in Solid State and Materials Science, Vol. 8, No. 1, 2004, pp. 31-37. http://dx.doi.org/10.1016/j.cossms.2003.10.006

[6] G.-X. Chen, H.-S. Kim, B. H. Park and J.-S. Yoon, "Multi-Walled Carbon Nanotubes Reinforced Nylon 6 Composites,” Polymer, Vol. 47, No. 13, 2006, pp. 4760-4767. http://dx.doi.org/10.1016/j.polymer.2006.04.020

[7] Q. Xiao, S. He, L. Liu, X. Guo, K. Shi, Z. Du, et al., "Coating of Multiwalled Carbon Nanotubes with Crosslinked Silicon-Containing Polymer,” Composites Science and Technology, Vol. 68, No. 1, 2008, pp. 321-328. http://dx.doi.org/10.1016/j.compscitech.2006.09.001

[8] Y. H. Xu, Q. F. Li, D. Sun, W. J. Zhang and G. X. Chen, "A Strategy To Functionalize the Carbon Nanotubes and the Nanocomposites Based on Poly(l-lactide),” Industrial \& Engineering Chemistry Research, Vol. 51, No. 42, 2012, pp. 13648-13654. http://dx.doi.org/10.1021/ie300989w

[9] C. Bartholome, P. Miaudet, A. Derré, M. Maugey, O. Roubeau, C. Zakri, et al., "Influence of Surface Functionalization on the Thermal and Electrical Properties of Nanotube-PVA Composites," Composites Science and Technology, Vol. 68, No. 12, 2008, pp. 2568-2573. 
http://dx.doi.org/10.1016/j.compscitech.2008.05.021

[10] R. D. Farahani, H. Dalir, V. Le Borgne, L. A. Gautier, M. A. El Khakani, M. Lévesque, et al., "Reinforcing Epoxy Nanocomposites with Functionalized Carbon Nanotubes via Biotin-Streptavidin Interactions,” Composites Science and Technology, Vol. 72, No. 12, 2012, pp. 1387-1395. http://dx.doi.org/10.1016/j.compscitech.2012.05.010

[11] X. Y. Gong, J. Liu, S. Baskaran, R. D. Voise and J. S. Young, "Surfactant-Assisted Processing of Carbon Nanotube/Polymer Composites,” Chemistry of Materials, Vol. 12, No. 4, 2000, pp. 1049-1052. http://dx.doi.org/10.1021/cm9906396

[12] C. Richard, F. Balavoine, P. Schultz, T. W. Ebbesen and C. Mioskowski, "Supramolecular Self-Assembly of Lipid Derivatives on Carbon Nanotubes,” Science, Vol. 300, No. 5620, 2003, pp. 775-778. http://dx.doi.org/10.1126/science.1080848

[13] Q. Wang, Y. Han, Y. Wang, Y. Qin and Z.-X. Guo, "Effect of Surfactant Structure on the Stability of Carbon Nanotubes in Aqueous Solution,” The Journal of Physical Chemistry B, Vol. 112, No. 24, 2008, pp. 7227-7233. http://dx.doi.org/10.1021/jp711816c

[14] M. N. Zhang, L. Su and L. Q. Mao, "Surfactant Functionalization of Carbon Nanotubes (CNTs) for Layer-byLayer Assembling of CNT Multi-Layer Films and Fabrication of Gold Nanoparticle/CNT Nanohybrid," Carbon, Vol. 44, No. 2, 2006, pp. 276-283. http://dx.doi.org/10.1016/j.carbon.2005.07.021

[15] H. J. Barraza, F. Pompeo, E. A. O’Rea and D. E. Resasco, "SWNT-Filled Thermoplastic and Elastomeric Composites Prepared by Miniemulsion Polymerization," Nano Letters, Vol. 2, No. 8, 2002, pp. 797-802. http://dx.doi.org/10.1021/nl0256208

[16] M. J. O'Connell, P. Boul, L. M. Ericson, C. Huffman, Y. Wang, E. Haroz, et al., "Reversible Water-Solubilization of Single-Walled Carbon Nanotubes by Polymer Wrapping," Chemical Physics Letters, Vol. 342, No. 3-4, 2001, pp. 265-271.

http://dx.doi.org/10.1016/S0009-2614(01)00490-0

[17] H. J. Li, X. B. Wang, Y. L. Song, Y. Q. Liu, Q. S. Li, L. Jiang, et al., "Super-Amphiphobic Aligned Carbon Nanotube Films," Angewandte Chemie International Edition, Vol. 40, No. 9, 2001, pp. 1743-1746. http://dx.doi.org/10.1002/1521-3773(20010504)40:9<174 3::AID-ANIE17430>3.0.CO;2-\#

[18] A. Kowalski, A. Duda and S. Penczek, "Kinetics and Mechanism of Cyclic Esters Polymerization Initiated with Tin(II) Octoate. 3.† Polymerization of 1,l-Dilactide," Macromolecules, Vol. 33, No. 20, 2000, pp. 7359-7370. http://dx.doi.org/10.1021/ma000125o

[19] V. Ambrogi, G. Gentile, C. Ducati, M. C. Oliva and C. Carfagna, "Multiwalled Carbon Nanotubes Functionalized with Maleated Poly(propylene) by a Dry Mechano-Chemical Process,” Polymer, Vol. 53, No. 2, 2012, pp. 291-299. http://dx.doi.org/10.1016/j.polymer.2011.11.048

[20] A. N. Chakoli, J. Wan, J. T. Feng, M. Amirian, J. H. Sui and W. Cai, "Functionalization of Multiwalled Carbon Nanotubes for Reinforcing of Poly(l-lactide-co-e-caprolactone) Biodegradable Copolymers," Applied Surface Science, Vol. 256, No. 1, 2009, pp. 170-177. http://dx.doi.org/10.1016/j.apsusc.2009.07.103

[21] J. Feng, W. Cai, J. Sui, Z. Li, J. Wan and A. N. Chakoli, "Poly(l-lactide) Brushes on Magnetic Multiwalled Carbon Nanotubes by In-Situ Ring-Opening Polymerization," Polymer, Vol. 49, No. 23, 2008, pp. 4989-4994. http://dx.doi.org/10.1016/j.polymer.2008.09.022

[22] H. X. Xu, X. B. Wang, Y. F. Zhang and S. Y. Liu, "Single-Step in Situ Preparation of Polymer-Grafted MultiWalled Carbon Nanotube Composites under 60Co ç-Ray Irradiation," Chem Mater Vol. 18, No. 13, 2006, pp. 2929-2934. http://dx.doi.org/10.1021/cm052840o

[23] S. M. Chen, G. Z. Wu, Y. D. Liu and D. W. Long, "Preparation of Poly(acrylic acid) Grafted Multiwalled Carbon Nanotubes by a Two-Step Irradiation Technique,” Macromolecules, Vol. 39, No. 1, 2006, pp. 330-334. http://dx.doi.org/10.1021/ma0520500

[24] J. Lunt, "Large-Scale Production, Properties and Commercial Applications of Polylactic Acid Polymers,” Polymer Degradation and Stability, Vol. 59, No. 1-3, 1998, pp. 145-152. http://dx.doi.org/10.1016/S0141-3910(97)00148-1

[25] R. E. Drumright, P. R. Gruber, D. E. Henton, "Polylactic Acid Technology,” Advanced Materials, Vol. 12, No. 23, 2000, pp. 1841-1846.

http://dx.doi.org/10.1002/1521-4095(200012)12:23<1841 $\because$ AID-ADMA1841>3.0.CO;2-E

[26] G.-X. Chen, H.-S. Kim, B. H. Park and J.-S. Yoon, “Controlled Functionalization of Multiwalled Carbon Nanotubes with Various Molecular-Weight Poly(l-lactic acid)," The Journal of Physical Chemistry B, Vol. 109, No. 47, 2005, pp. 22237-22243. http://dx.doi.org/10.1021/jp054768n

[27] G.-X. Chen, H.-S. Kim, B. H. Park and J.-S. Yoon, "Synthesis of Poly(L-lactide)-Functionalized Multiwalled Carbon Nanotubes by Ring-Opening Polymerization," Macromolecular Chemistry and Physics, Vol. 208, No. 4, 2007, pp. 389-398.

http://dx.doi.org/10.1002/macp.200600411

[28] W. Song, Z. Zheng, W. Tang and X. Wang, “A Facile Approach to Covalently Functionalized Carbon Nanotubes with Biocompatible Polymer," Polymer, Vol. 48, No. 13, 2007, pp. 3658-3663. http://dx.doi.org/10.1016/j.polymer.2007.04.071

[29] J. T. Yoon, S. C. Lee and Y. G. Jeong, "Effects of Grafted Chain Length on Mechanical and Electrical Properties of Nanocomposites Containing Polylactide-Grafted Carbon Nanotubes," Composites Science and Technology, Vol. 70, No. 5, 2010, pp. 776-782.

http://dx.doi.org/10.1016/j.compscitech.2010.01.011 